\title{
UNA ALTERNATIVA A LA EDUCACIÓN TRADICIONAL: EL APRENDIZAJE COOPERATIVO
}

Ramón Ferreiro Gravié

\section{INTRODUCCIÓN}

Cada época histórica ha tenido su propuesta educativa para las nuevas generaciones en respuesta a las condiciones y exigencias sociales, económicas, políticas e ideológicas del momento.

El siglo XX fue un período de grandes logros en el campo educativo. Pocas veces, en un lapso de tiempo semejante, han existido tantos aportes científicos pedagógicos, en el plano teórico pero también metodológico y práctico.

Los diferentes paradigmas psicopedagógicos surgidos en el decursar del siglo XX han conformado diferentes propuestas educativas a partir de sus presupuestos teóricos ${ }^{1}$.

Así, el paradigma conductista propuso la tecnología educativa y la enseñanza programada que constituyeron, en su momento, una verdadera revolución en la enseñanza.

Por su parte, el paradigma humanista planteó la educación personalizada, y el movimiento de orientación cognoscitivista aportó los proyectos y programas de enseñar a pensar, y aprender a aprender.

El paradigma sociocultural o sociohistórico, como también se le nombra, enfatizó, dada su concepción teórica, la necesidad de una educación desarrolladora y una enseñanza problémica.

En los últimos años del siglo pasado, la concepción constructivista, más específicamente hablando, el constructivismo social posee, entre sus propuestas educativas másinnovadoras, al aprendizaje cooperativo.

Al aprendizaje cooperativo también se le conoce como

${ }^{1}$ HERnÁNDEZ G., Paradigmas en Psicología de la Educación., Ed. Paidós Ecuador., México., 1998., pp.36-37. 
aprendizaje entre iguales o aprendizaje entre colegas, a partir del criterio de que "el mejor maestro de un niño es otro niño".

Otros especialistas le llaman aprendizaje colaborativo, pero es bueno recordar la diferencia existente entre colaboración y cooperación. Al menos en nuestro idioma, la diferencia conceptual es destacable.

La enseñanza cooperativa o bien educación cooperativa es empleada con menos frecuencia, quizás por la necesidad de distanciar conceptualmente esta alternativa educativa de los planteamientos de John Dewey (1859-1952) y Celestin Freinet (1896-1966) al principio de siglo XX, que aunque válidos como antecedentes, la noción y práctica de aprendizaje cooperativo de nuestros días, dista mucho de la concepción pedagógica de los destacados científicos de la educación que se inscribían en el movimiento de la Escuela Nueva ${ }^{2}$.

Otros autores, los menos, hablan de aprendizaje en equipo, pero esta denominación por lo regular se evita por la imagen que puede provocar, en muchos, que aprendizaje cooperativo es simplemente aprendizaje grupal.

Si bien el aprendizaje cooperativo es aprendizaje en grupos, también es mucho más, como también es mucho más que lo planteado por la Escuela Nueva en su momento.

Las ideas pedagógicas esenciales del aprendizaje cooperativo no son nuevas (Johnson, D. W. 1999., Ovejero, A. 1989), han estado presentes a lo largo de la historia de la educación; lo que es nuevo es la reconceptualización teórica hecha a partir de los puntos de vista de la ciencia contemporánea y las investigaciones experimentales e investigación acción, así como su eficacia en la práctica educativa actual, en comparación con otras formas de organizar el proceso de enseñanza.

\footnotetext{
${ }^{2}$ Expresión con que se identifica un movimiento pedagógico de finales del siglo XIX y parte del siglo XX, caracterizado entre otras cosas por el énfasis en la necesidad de la actividad, la libertad, la individualidad y la colectividad en la educación infantil, además de su marcado enfoque paidocéntrico, a diferencia de las corrientes pedagógicas que asignaban al educador todo el esfuerzo y orientación del proceso educativo. Santillana. Diccionario de las Ciencias de la Educación. Santillana, México, 1990, p.574.
} 
El propósito de este trabajo es destacar los aspectos básicos del aprendizaje cooperativo como "nueva" forma de organización del proceso educativo, que lo distinguen de sus antecedentes, fuentes y variantes contemporáneas. En otras palabras, responder a la pregunta: ¿qué lo hace ser un modelo educativo innovador? ¿Cuál es su $a b c$ ?

\section{UN ASPECTO SIN DISCUSIÓN: LA PARTICIPACIÓN}

La $a$ del $a b c$ del aprendizaje cooperativo se refiere a la actividad, a la forma peculiar y distintiva del aprendizaje cooperativo de hacer participar a los alumnos en su proceso de aprendizaje.

El énfasis está en la necesidad de la participación del sujeto que aprende, en su proceso de aprendizaje, su actividad externa, pero también interna, es decir aquélla referida a los procesos psicológicos superiores que provoca la actividad externa; más aún, la necesidad de tener en cuenta los procesos de comunicación inherente a toda actividad humana.

Y he aquí una diferencia sustancial del aprendizaje cooperativo con los modelos educativos que le precedieron: la participación que toma en cuenta la unidad entre la actividad interna y externa, y más aún entre la actividad y la comunicación.

De Jean Piaget (1896-1980) se toma, por así decirlo, toda la fundamentación teórica de la organización de situaciones de aprendizaje en que se da el enfrentamiento del sujeto que aprende, con el objeto de conocimiento; a esos momentos se les llama interactividad.

Para aprender, es necesaria esa confrontación con el objeto de aprendizaje, es decir, con el contenido de enseñanza.

Pero para aprender significativamente se requieren además, momentos de interacción del sujeto que aprende con otros, que le ayuden a moverse de un "no saber, a saber", de "no poder hacer, a saber hacer" y, lo que es más importante, de "no ser, a ser" (Vigotsky, 1997).

Lo antes planteado es precisamente lo que el aprendizaje 
cooperativo toma de la teoría de Lev S. Vigotsky (1896-1934): la necesidad del otro, de las otras personas para aprender significativamente.

La participación genuina, la verdadera actividad de los alumnos en clase, exige de momentos de interactividad y momentos de interacciones como una unidad, como dos caras de una misma moneda.

De ahí que la fórmula para expresar gráficamente el aprendizaje cooperativo, sea igual a momentos de trabajo individual, equivalentes a la interactividad necesaria para aprender, y de momentos de trabajo con otros, identificados con los procesos de interacciones entre los sujetos que aprenden: ni todo el tiempo en solitario, ni todo el tiempo en grupo. La concepción del aprendizaje cooperativo exige de ambos momentos los cuales, si sabemos alternarlos didácticamente, optimizan el esfuerzo individual y también el del trabajo en equipos.

Otra peculiaridad distinta del aprendizaje cooperativo -con relación a las propuestas educativas del movimiento de la Escuela Nueva y del llamado aprendizaje dinámico o activo, así como de la dinámica de grupo derivada de la teoría de los grupos operativos de Enrique Pichón Riviere (1907-1977)- es que, si bien es necesario incrementar la participación de los alumnos en su proceso de aprendizaje, también es imprescindible diversificar las formas de hacerlo, de manera tal que se estimulen las diferentes áreas neuropsicológicas "comprometidas" y provoquen un aprendizaje con todo el cerebro y por tanto un desarrollo pleno de la persona.

Esto último no lo toman en consideración las alternativas educativas antes enunciadas, por lo relativamente reciente de las investigaciones sobre el cerebro que tienen, entre otros hitos, los planteamientos del doctor Robert W. Sperry (que en 1981 se hizo merecedor del Premio Nobel de Medicina, al demostrar la especificidad funcional de los hemisferios cerebrales, y justificar que el cerebro izquierdo es responsable del desarrollo del pensamiento lógico, crítico, secuencial y analítico, 
y que el cerebro derecho lo es del pensamiento creativo, intuitivo, sintético y holístico $)^{3}$.

Otro hito importante, fueron, sin duda, los planteamientos del doctor Norbert Hermann sobre la existencia de "cuatro cerebros", demostrándose la mayor especificidad de cada uno de los dos cerebros, derecho e izquierdo, ya mencionados, y su propuesta de una tipología comportamental inspirada, entre otros, en los trabajos de Sperry (Chalvin, M. J., 1995).

Lo más importante de las investigaciones de $R$. Sperry y de $N$. Hermann son sus implicaciones para la práctica educativa, que la didáctica del aprendizaje cooperativo hace suyas con la finalidad de incrementar, y lo que es más importante, diversificar la participación de los alumnos en clase, de forma tal que se aprovechen sus extraordinarias potencialidades para aprender y, a su vez, favorecer su desarrollo integral.

En resumen, la $a$ del $a b c$ del aprendizaje cooperativo tiene que ver con la necesidad de la actividad del alumno en clase, nunca espontánea, en todo instante orientada, guiada e intencionada, pero con una libertad responsable y comprometida de los aprendices.

\section{EXIGENCIA CLAVE: MEDIACIÓN PEDAGÓGICA}

La $b$ del $a b c$ del aprendizaje cooperativo se relaciona con la bidireccionalidad necesaria en el proceso de aprendizajeenseñanza, entre el que guía y orienta la actividad, y el aprendiz.

El aprendizaje cooperativo plantea una forma diferente de relacionarse entre el maestro y el alumno y los alumnos entre sí, en su proceso de aprender. Ese modo de relacionarse es la mediación.

Al respecto, el filósofo alemán Hegel en más de unas de sus obras, se refiere a la mediación, y el psicólogo ruso Vigotsky introduce este concepto en la literatura psicológica

${ }^{3}$ Para profundizar en los aportes de las neurociencias a la educación, se puede consultar entre otras la obra de CHALVIN, Marie Joseph, Los dos cerebros en el aula. Conocer la dominancia cerebral para mejorar la educación. 
al explicar el papel del otro en su concepto de zona de desarrollo potencial o próxima ${ }^{4}$.

Georg Wilhelm Friedrich Hegel (1770-1831) aportó una teoría dialéctica del desarrollo que todavía hoy es un punto de referencia para comprender la realidad, y como parte de ella, el papel del sujeto mediador y del proceso de mediación para conocer el mundo que nos rodea.

Lev Semiónovich Vigotsky (1896-1934) tomó de Hegel el concepto de mediación y lo introdujo en la literatura psicológica como un componente medular para explicar el tipo de relación entre un adulto que sabe y puede realizar una tarea, y otro sujeto que requiere de ayuda para hacerlo en el marco conceptual de la zona de desarrollo potencial.

Vigotsky plantea la existencia de dos niveles evolutivos. Al primero le llama nivel real y lo identifica con el grado de desarrollo psicológico que presenta el niño en un momento dado.

El nivel real de desarrollo es el resultado de los procesos evolutivos cumplidos y es el que precisamente manifiesta al aplicar tests psicológicos o pruebas pedagógicas estandarizadas.

El otro nivel evolutivo, el potencial, se hace patente ante una tarea que el niño no puede solucionar por sí solo, pero que es capaz de realizar si recibe ayuda de un adulto, papás, maestros o bien de un compañero más capaz.

Y es precisamente a este tipo de relación -mejor aún, interacción social, adulto-niño-, que estimula el desarrollo de las potencialidades del sujeto, la que Vigotsky conoce como mediación educativa, y al sujeto portador de la experiencia como mediador.

Vigotsky argumenta que el origen de la concepción del mundo está en las primeras experiencias de aprendizaje originadas con las personas más diestras que nosotros y que nos

\footnotetext{
${ }^{4}$ Leóntiev, A. N., amigo y cercano colaborador de Vigotsky, plantea que: "La zona de desarrollo próximo caracteriza la diferencia entre lo que el niño es capaz de alcanzar por su cuenta y lo que es capaz de conseguir con ayuda del instructor" L. S. Vigotsky., Obras Escogidas. Tomo I, Ed. Aprendizaje Visor, Madrid., España., 1997, p.445.
} 
ayudan a encontrarle el sentido y significado a los objetos y eventos de la realidad.

Si bien es cierto que el aprendizaje directo posee su valor, también lo es que el aprendizaje mediado es condición y fuente del buen aprendizaje directo dado, entre otras cosas, por su contribución a que el sujeto le encuentre sentido y significado al objeto de conocimiento.

Al respecto Vigotsky plantea: "Cada función psíquica aparece en el proceso de desarrollo de la conducta dos veces; primero, como función de la conducta colectiva, como forma de colaboración o interacción, como medio de la adaptación social o sea, como categoría interpsicológica, y, en segundo lugar, como modo de la conducta individual del niño, como medio de adaptación personal, como proceso interior de la conducta, es decir, como categoría intrapsicológica " 5 .

En los últimos años, un psicólogo israelita de origen rumano, Reuven Feuerstein (1921- ) retoma la concepción vigotskiana de sujeto mediador y de proceso de mediación, y la incorpora como elemento sustantivo de una de sus dos teorías: la teoría de la experiencia de aprendizaje mediado y de sus tres propuestas prácticas ${ }^{6}$.

El mediador es la persona que al relacionarse con otro o con otras:

- Favorece su aprendizaje.

- Estimula el desarrollo de sus potencialidades.

- Y lo que es más importante: corrige funciones cognitivas deficientes.

Los abuelos, padres, tíos, hermanos mayores, amigos pueden ser mediadores. En general todo aquel que se relaciona

\footnotetext{
${ }_{5}^{5}$ VIGOTSKI, L.S. La colectividad como factor de desarrollo del niño deficiente., Obras completas., Tomo V., Ed. Aprendizaje Visor., Madrid., España., 1997., p.214.

${ }^{6}$ Feuerstein, R. ha aportado dos teorías: la modificabilidad estructural cognitiva y la experiencia de aprendizaje mediado. Así como tres programas o propuestas prácticas: el programa de enriquecimiento instrumental (PEI); el programa de la evaluación dinámica del potencial de aprendizaje (LPAD); y el más reciente, el de la creación de ambientes enriquecidos de aprendizaje.
} 
con otros y, al hacerlo, cumple con determinados requisitos es mediador.

Los maestros son o deben ser mediadores por excelencia.

Según Reuven Feuerstein, al mediar, el maestro debe cumplir con ciertos requisitos. Entre otros ${ }^{7}$, los más importantes son:

- La reciprocidad: una relación actividad-comunicación mutua, en la que ambos, mediador y alumno, participan activamente.

- La intencionalidad: tener bien claro qué quiere lograr y cómo ha de lograrse, tanto uno, el maestro mediador, como el alumno que hace suya esa intención dada la reciprocidad que se alcanza.

- El significado: que el alumno le encuentre sentido a la tarea.

- La trascendencia: equivale a ir más allá del aquí y el ahora, y crear un nuevo sistema de necesidades que muevan a acciones posteriores.

- El sentimiento de capacidad o autoestima: o lo que es lo mismo, despertar en los alumnos el sentimiento de que son capaces.

En resumen, la $b$ del $a b c$ del aprendizaje cooperativo se refiere a la bidireccionalidad que debe caracterizar el proceso de aprendizaje-enseñanza, es decir a la reciprocidad intencionada que se logra gracias a la mediación.

Todo proceso de mediación se basa en la premisa de que es posible la modificabilidad de las estructuras cognitivas ${ }^{8}$ y también afectivas del sujeto que aprende y que éstas se propician con una dirección de la enseñanza de tipo no frontal.

7 MARTÍNEZ, J. MA., Modificabilidad cognitiva y programa de enriquecimiento instrumental, Ed. Instituto Superior., S. Pío X., Madrid., España., p.5-7.

${ }^{8} \mathrm{El}$ concepto de modificabilidad, se refiere a los cambios en la manera en que el organismo humano se interrelaciona, es decir, actúa y responde a las fuentes de información, determinando el curso de desarrollo de un individuo. Se parte de la premisa de que la conducta de la persona humana es susceptible de modificarse a partir de una intención educativa y con estrategias adecuadas para ello dado, entre otros factores, el de las características de su sistema nervioso. 


\section{CONDICIÓN BÁSICA: LA COOPERACIÓN ENTRE IGUALES}

La $c$ del $a b c$ del aprendizaje cooperativo, alude a la cooperación entre las personas para aprender en clase.

Según Roger y David Johnson de la Universidad de Minnesota, son tres las formas de relación entre los alumnos para aprender: la individualista, la de competencia y la de cooperación

En la individualista, cada uno se ocupa de lo suyo sin importarle el otro. No interesa que uno y otros se comuniquen entre sí e intercambien sobre lo que se aprende. La distribución frontal del salón y el método expositivo del maestro se justifica por este modo de concebir la enseñanza.

Otra forma es la competitiva, que se observa cuando cada uno de los miembros de un grupo escolar percibe que pueden obtener el objetivo de enseñanza, si, y sólo si, los restantes alumnos no lo obtienen.

El tercer tipo básico de relación para aprender es la cooperación, que nace cuando cada uno de los que integran el equipo percibe que puede lograr el objetivo si, y sólo si, todos trabajan juntos y cada cual aporta su parte.

Cooperar es compartir una experiencia vital significativa que exige trabajar juntos para lograr beneficios mutuos.

La cooperación implica resultados en conjunto, mediante una interdependencia positiva que involucra a todos los miembros del equipo en lo que se hace, y en el proceso del cual, cada uno aporta su talento.

La cooperación entre los miembros de un grupo escolar es la piedra angular de esta forma de organización del proceso de enseñanza: el aprendizaje entre iguales o entre colegas.

La relación, mejor aún, la interrelación cooperativa entre los compañeros en clase les aporta, entre otras cosas:

- Modelos a imitar.

- Oportunidades de hacer, decir y sentir.

${ }^{9}$ JOHNSON, D. W. et al., Los nuevos círculos de aprendizaje. Cooperación en el salón de clase y en la escuela. Ed. ASCD., Virginia, EEUU., 1995., p.1-3. 
- Apoyo según necesidades manifiestas.

- Expectativas in crescendo.

- Autorregulación personal y en equipo.

- Refuerzo positivo constante.

- Perspectivas diferentes sobre un mismo asunto.

- Desarrollo de las habilidades cognitivas pero también sociales y afectivas.

\section{CONCLUSIÓN}

Son muchas las razones que, a partir de los resultados de investigaciones experimentales, de investigaciones acción y de la sistematización de la práctica de los maestros que lo aplican, justifican el aprendizaje cooperativo como una alternativa novedosa necesaria para resolver las exigencias educativas de la sociedad actual. Entre las ventajas del aprendizaje cooperativo destacamos, en apretada síntesis:

1. Cualifica la participación de los alumnos en su proceso de aprendizaje escolar, dado que además de incrementar su actividad-comunicación, la diversifica, haciendo "uso" tanto del cerebro izquierdo como del derecho.

2. Plantea una dirección no frontal, mediatizada, del proceso de enseñanza escolar que favorece el desarrollo de las potencialidades del sujeto que aprende, en particular su autonomía personal y social.

3. Establece un tipo de relación de cooperación entre los alumnos, estimulando su desarrollo cognitivo y socio afectivo, lo que resulta imprescindible para el aprendizaje de actitudes y valores.

Más aún, la aplicación de la concepción didáctica del aprendizaje cooperativo es condición necesaria, aunque por supuesto no suficiente, para la aplicación con éxito de otras novedosas alternativas educativas como son el estudio de caso, el método de proyecto, la enseñanza basada en problemas, el método de contrato y los talleres vivenciales, así como el empleo de las tecnologías de punta en los nuevos ambientes de aprendizaje. 


\section{BIBLIOGRAFIA}

CALDERÓN, M. Teachers learning communities for cooperation in diverse settings. Theory Into Practice., Volumen 38., Number 2., The Ohio State University. Spring 1999., 116 p.

COHEN, E. et al. Complex instruction: equity in cooperative learning classrooms. Theory Into Practice. Volumen 38., Number 2., The Ohio State University Spring 1999, 116 p.

COLL, C. et al. Aprendizaje escolar y construcción del conocimiento., Cap. 5., "Estructura grupal, interacciones entre alumnos y aprendizaje escolar"., Ed. Paidós Educador., México., 1977., 206 p. COSTA, A. L. et al. Cognitive Coaching., A foundation of renaissance schools. Chistopher Gordon Publishers. Inc. Norwood, Ma., E.E.U.U., 1994., 241 p.

CUAdernos DE PEDAGOGíA. Pedagogías del siglo XX. Ed. Cisspraxis, S.A. Barcelona., España., 2000., 159 p.

CHALVIN, M. J., Los dos cerebros en el aula., Ediciones TEA., Madrid., España., 1995., 200 p.

HERNÁNDEZ, G., Paradigmas en Psicología de la Educación., Ed. Paidós Educador., México., 1998., 267 p.

FERREIRO, R. et al. El ABC del aprendizaje cooperativo., Ed. Trillas., México., 2000., 125 p.

FERREIRO, R. La mediación pedagógica: exigencia clave en la escuela del siglo XXI., Revista Educación 2001., México., abril 2002., 80 p FEUERSTEIN, R. Et al. Mediated learning in and out of the classroom., Ed. Iris/Skylight., Training and Publishing., Inc., Illinois., E.E.U.U., 1996., 186 p.

HERTZ-LAZAROWITZ, R., Cooperative learning in Israel's jewish and arab schools: A community approach. Theory Into Practice., Volumen 38., Number 2., The Ohio State University., Spring 1999., $116 \mathrm{p}$.

JOHNSON, D. W. et al. Los nuevos círculos de aprendizaje. Cooperación en el salón de clases y en la escuela., Ed. Asociación para la supervisión y desarrollo de programas de estudio., Virginia., E.E.U.U.., 1995., 117 p. 
JOHNSON D. W.. et al. Making Cooperative work. Theory Into Practice., Volumen 38., Number 2., The Ohio State University., Spring., 1999., $116 \mathrm{p}$.

LOBATO, C., El trabajo en grupo. Aprendizaje cooperativo en secundaria., Servicio Editorial Universidad del País Vasco., España., 1998., 114 p.

KAGAN, S., Cooperative learning., Ed. Kagan Cooperative learning., San Juan Capistrano., Ca., E.E.U.U., 1993., 2413 p.

MEIER, Ben-Hur. et al. On Feuerstein Instrumetal Enrichment., A collection., Ed. Iris/Skylight., Training and publisching., Inc., Illinois., E.E.U.U., 1998., 282 p.

OVEJERO, A., El aprendizaje cooperativo: Una alternativa eficaz a la enseñanza tradicional., Oviedo., España., 1989., 334 p.

RUÉ, Joan., El treball cooperatiu. L'organització social de l'ensenyament $i$ l'aprenentatge., Ed. Barcanova Educación., Barcelona., España., 1991., 219 p.

SHARAN, Sh., Editor. et al. Handbook of cooperative learning methods., Greenwood Publishing Group., Inc., E.E.U.U., 1994., $350 \mathrm{p}$.

SLAVIN, R. et al. Effective programs for latino students., Laurence Erlbaum Associates, Publishers., New Jersey., E.E.U.U., London., U.K., 2000., 394 p.

SLAVIN, R. et al. Every child, every school. Success for all., Corwin Press., Inc., California., E.E.U.U., 1996., 246 p.

STAHL, R. J. Editor. et al. Cooperative learning in language arts. A handbook for teachers., Ed. Innovative Learning Publications., Ca., E.E.U.U., 1995., 430 p.

VYGOTSKI, L. S., Obras escogidas., Tomo I., Ed. Aprendizaje Visor., Madrid., España., Segunda Edición., 1997., 496 p.

VYGOTSKI, L. S. Obras escogidas., Tomo III., Ed. Aprendizaje Visor., Madrid., España., 1995., 383 p.

VYGOTSKI, L. S. Obras escogidas., Tomo V., Ed. Aprendizaje Visor., Madrid., España., 1997., 391 p. 